\title{
DE LA SITUACIÓN PROBLEMÁTICA AL PROBLEMA CIENTÍFICO EDUCACIONAL
}

\author{
Felipe Aguirre Chávez*
}

\section{Introducción}

Los problemas científicos de las investigaciones educacionales emergen del conocimiento del estado actual de desarrollo del tema que se pretende investigar. La primera etapa es el diagnóstico, la aplicación de instrumentos, con fines exploratorios, como las entrevistas, encuestas, observaciones, etc.; donde el investigador, independientemente de su objeto de estudio, desentraña, establece o determina situaciones problemáticas con sus perspectivas de solución. Metafóricamente hablando, toda situación problemática viene con la formulación del problema bajo el brazo; pero configurarlo como problema científico viable, novedoso y pertinente requiere motivación y destrezas específicas de problematización en sus múltiples perspectivas de solución.

En dependencia del enfoque y las habilidades del investigador, existen hasta dos posibilidades básicas: pasar con éxito de la situación problemática a la formulación del problema científico o quedar anclado en el callejón sin salida de la imposibilidad de problematizar; y, por ende, de rezagar la producción de nuevos conocimientos.

En el presente artículo defendemos la idea de que la situación problemática y formulación del problema científico, constituyen aspectos diferentes; pero relacionados. El desarrollo adecuado del diagnóstico permitirá determinar la situación problemática y el problema científico; en perspectiva, también, conocer si es pertinente, viable, novedoso y relevante; o, por el contrario, es espurio.

En ese contexto es importante reflexionar sobre el primer momento metodológico de la investigación científica educacional: El problema, ¿qué es la situación problemática?, ¿cómo se determina la existencia de la situación problemática?, ¿de qué tipo es la situación problemática?, ¿qué relación existe entre situación problemática y problema científico? y finalmente ¿qué es el problema científico?

\footnotetext{
* AGUIRRE CHAVEZ, FELIPE trabaja como docente en las Escuelas de Posgrado de la Universidad Nacional de Educación, Universidad Champagnat y Universidad San Ignacio de Loyola. Trabajó como especialista en el Ministerio de Educación del Perú y participó como auditor educativo-Contraloría General de la República. Autor de textos, artículos científicos y proyectos de innovación que han recibido premios nacionales (2002, 2004,2007 y 2012).
} 


\section{El problema}

El significado etimológico de la palabra problema proviene del griego probhma, próblema, «lo puesto delante», del verbo proballo, «poner delante». En el sentido primigenio, el problema emerge, se manifiesta como obstáculo que podrían tener múltiples manifestaciones: dificultades, dudas, disgustos, preocupaciones, vacíos cognoscitivos, anomalías, conflictos cognitivos o contradicciones.

Según el DRAE (2005), la palabra problema está referida al planteamiento de una situación cuya respuesta desconocida debe obtenerse a través de métodos científicos. Situación que, según Dewey, citado por Kerlinker (2002, p. 14), se precisa como "situación problemática, perpleja, irritante, donde la dificultad se encuentra a todo lo largo y ancho de ella, afectándola como un todo".

Bunge (1975) refiere que el problema designa una dificultad que no puede resolverse automáticamente; sino que requiere una investigación. Así, un problema viene a ser el primer eslabón de una cadena: problema-investigación-solución.

Desde el enfoque de la investigación educacional, según Lanuez, Martínez y Pérez (20l0) "el problema es la manifestación de una situación problemática, la que a su vez es expresión de una contradicción entre el estado deseado y el real de una situación teórica o práctica" (p. 58). Según esta definición, el problema es, en lo fundamental, lo conocido de lo desconocido. Como indican Campistrous y Rizo (1996), toda situación en la que hay un planteamiento inicial y una exigencia que obliga a transformarlo. El desconocimiento de la solución indica que hay problema. Es decir, la vía para pasar de la situación o planteamiento inicial a la nueva situación exigida, eventualmente, se desconoce; cuando es conocida deja de ser problema.

Observar la realidad con valoración crítica creadora, encontrar problemas donde los demás no lo logran, constituye el resultado interactivo múltiple de destrezas cognitivas; por lo mismo, constituye mérito científico, capacidad de encontrar anomalías o vacíos cognoscitivos donde otros no ven o encuentran problemas. Sin temor a equivocación, se requiere comprensión metodológica, inspiración creadora, estudio sistemático, persistencia y motivación tanto en el proceso como en el resultado problematizador proyectivo de probables soluciones científicas.

\section{2. ¿Qué es la situación problemática?}

La situación problemática tiene dos términos que, metodológicamente, son importantes definir por separado. Primero el significado de "situación" que, en términos generales, según la DRAE (2009) son el conjunto de factores o circunstancias que afectan a alguien o algo en un determinado momento. 
La realidad y sus necesidades, al ser infinitas, requieren ser delimitadas, situadas en un lugar, en un estado de desarrollo de tiempo y espacio. Situada el objeto de estudio es más viable caracterizarlo. Al respecto, el Manual de publicaciones de la APA (20 I0) refiere que "como regla general, describa los grupos de la manera más específica posible..." (p. 29). Asimismo, autores como García y García (20 I0) mencionan que la situación problemática debe ser concreta y delimitada. Con ello, defendemos la idea de que las investigaciones educacionales, primordialmente, asumen situaciones problemáticas específicas de la práctica educativa, pedagógica o didáctica; por cuanto, la novedad y utilidad de la investigación educacional consiste en encontrar soluciones científicamente fundamentadas a los problemas del proceso educativo o de enseñanza aprendizaje.

El otro término es el de "problemática", que, según Hernández (2009), es "algo dudoso, poco probable, que no es cierto, inconstante, desconocido o ignorado, cuestionable e indiscutible; en fin, caótico" (p. 2). Por su parte, Lanuez et al. (20 I0) indican que lo problemático "es un estado de desigualdad, contradicción... o necesidad educativa" (p. 57).

En suma, la situación problemática constituye factores o circunstancias contradictorias de vacíos cognoscitivos o de obstáculo con existencia real e independencia del sujeto; pero con afección por el que se interesa en comprenderlo y, eventualmente, resolverlo.

\section{3. ¿Cómo se determina la existencia de la situación problemática?}

La situación problemática es el resultado de comparar el comportamiento real con el comportamiento ideal. Si ambos comportamientos son iguales, no existe contradicción o situación problemática; si, por el contrario, se presenta un estado de desigualdad, entonces hay situación problemática.

El comportamiento ideal es el conjunto de potencialidades: el valor, la importancia teórica, metodológica, establecida en la literatura general o específica a nivel de enfoques y teorías. Un ejemplo típico es que los niños de segundo grado deben leer comprensivamente sesenta palabras por minuto. Eso es lo ideal, lo que esperamos, pero en muchas ocasiones dista de la realidad. Entonces hablamos de comportamiento real como dificultades, necesidades, vacíos cognoscitivos u obstáculos situados en un contexto específico. Siguiendo el ejemplo anterior, los niños de segundo grado de primaria de un contexto específico leen sin comprensión apenas 20 palabras por minuto.

Otro ejemplo, si observamos que los alumnos de un grupo no cuidan adecuadamente el medio ambiente; ése es el comportamiento real, sin embargo, esos alumnos, de acuerdo con su edad, ya tienen conocimientos para cuidar el medio, y lo deben 
hacer, este es el comportamiento deseado, es decir, lo deben hacer, pero no lo hacen; he ahí la contradicción, la situación problemática.

Al comparar los dos estados, si hay brecha, hay contradicción y si es así, estamos frente a una situación problemática. Como refiere Ron (1992), es una situación donde un individuo o grupo percibe una diferencia entre un estado presente y un estado deseado; diferencia, que según Labarrere y Valdivia (2005) se denomina "contradicción" (p. 19); es decir, situación problemática. El hallazgo indica que vale el esfuerzo de continuar con el resto del diseño teórico-metodológico de la investigación.

Metodológicamente, para determinar la situación problemática, según Launez et al., (2010), es necesario preguntarse: ¿qué necesitamos conocer?, ¿cómo lograr conocer lo que necesitamos? (p. 46). Así, si pretendemos estudiar dentro de la educación ambiental, el cuidado del medio ambiente por parte de los niños de cuarto grado de educación primaria; el investigador podría plantear dos cuestiones:

\section{¿Qué necesitamos conocer?}

- Si los alumnos, actualmente, en su quehacer diario, cuidan el medio ambiente.

\section{¿Cómo lograrlo?}

- Observando su comportamiento en las aulas y fuera de ellas.

- Creando situaciones de contacto directo con la naturaleza, como excursiones ambientalistas, y observando su comportamiento.

- Entrevistando a algunos niños.

- Entrevistando o encuestando a los padres de algunos de ellos.

- Entrevistando a los profesores que trabajan con los niños.

- Otras formas que decida el investigador.

Para logarlo, el investigador tiene hasta tres vías. La primera asumir instrumentos estandarizados; la segunda, trabajar en la contextualización de instrumentos; y la tercera, construir sus propios instrumentos confiables y válidos. Cualesquiera de las tres vías son importantes y deberán concretarse en el recojo de datos, resultados que indicarán si realmente estos niños cuidan el medio ambiente. Es decir, se determina el comportamiento real, el estado actual de desarrollo, del objeto o fenómeno de investigación. 


\section{4. ¿De qué tipo puede ser la situación problemática?}

Una vez detectado la situación problemática es necesario preguntarse de qué tipo es la situación problemática. Según García y García (20 l 0) una dificultad podría ser "teórica o práctica... un obstáculo o un vacío de información" (p. 5).

Las dificultades teóricas se identifican con los vacíos de conocimiento sobre determinados factores de la realidad, ese vacío cognoscitivo se superaría con investigaciones o "reinvestigaciones" que acopien información para la producción de nuevos conocimientos descriptivos y explicativos. Por ejemplo, desconocer los factores por los cuales los estudiantes se comportan de una manera determinada, no disponer de información para explicar las deficiencias en comprensión lectora o que no existan definiciones claras sobre el desempeño docente son algunos ejemplos de vacíos de información.

Las dificultades prácticas, requieren intervención o diseño de resultados científicos fundados en teorías que, al ser aplicadas eventualmente, permitirían la transformación del problema. Algunos ejemplos de problemas prácticos, según García y García (20 |4), son "el que una estrategia didáctica no promueva un aprendizaje significativo; una comunidad educativa no se desarrolle como es habitual, o un método de enseñanza no genere los resultados deseados" (p. 6).

En todo caso, identificar de qué tipo es la situación problemática, ayudará en la elección del tratamiento. Los obstáculos requerirán intervención transformadora sobre la realidad, sea para resolver una situación conflictiva, o bien para crear una situación nueva. En cambio, los problemas de vacíos de información requieren búsqueda de información.

Dicho de otro modo, cuando se busca diseñar planes de acción (modelo, estrategia, metodología, cuerpo de recomendaciones, entre otros), que posibiliten transitar al objeto de investigación del comportamiento real al deseado, son investigaciones de intervención transformadora; y, cuando se busca acopiar información descriptiva, correlacional, estamos frente a una investigación de conocimiento o "reinvestigación".

Desde luego, desde nuestra modesta forma de ver, considerando que existe, información amplia y relevante, sea exploratoria, descriptiva, correlacional y explicativa; las investigaciones educacionales deberían ser propositivas, de diseño de resultados científicos; que surjan de la práctica con base y fundamento, para regresar a la práctica.

\section{El problema científico.}

El problema científico, metafóricamente hablando, es la punta del iceberg de la 
problematización, es la síntesis de un proceso analítico y sintético, crítico y creativo a la vez.

En ocasiones, se confunde la situación problemática con el problema científico. Según el enfoque asumido, son dos conceptos con muchos puntos de contacto, pero no identificados totalmente.

Distinguir ambas categorías es un adelanto significativo en la formulación del problema científico. Concordando con Lanuez et al. (2010), "cuando se logra la formulación adecuada del problema, se ha logrado un gran avance, una sólida base para iniciar la investigación, y muchas probabilidades de obtener éxitos" (p. 55). En cualquier campo de investigación, es un imperativo categórico formular adecuadamente el problema, de él depende todo el accionar investigativo posterior, y en buena medida el éxito del proyecto investigativo. Como refiere Ackoff (1 967) “... un problema bien planteado es la mitad de la solución” citado por Lanuez y otros (20 l 0, p.55).

La adecuada formulación del problema ocurre en simultáneo con la fundamentación teórica, metodológica o práctica; y las posibilidades de solución. Por cuanto, a mayor conocimiento y experiencia sobre el tema, mayores probabilidades de problematizar y crear soluciones.

Las modalidades de formulación de problemas no son únicas, dependerá del enfoque, de la naturaleza de la situación problemática y de los objetivos que persigue el investigador. Aunque no existen reglas aceptadas unánimemente para la formulación de problemas científicos; siguen siendo válidos y, ampliamente, aceptados las orientaciones y criterios dados por Kerlinger.

Este último recomienda que la formulación del problema científico debe ser consecuencia de la identificación de una situación problemática y la mejor forma es la forma más simple: Elabore una pregunta (Kerlinger, 1964).

Según Kerlinger, se debe tener en cuenta tres criterios para la formulación de buenos problemas de investigación:

I. El problema deberá expresar una relación entre dos o más variables.

2. El problema debe formularse claramente y sin ambigüedades.

3. El problema y la formulación del mismo deben ser tales que impliquen las posibilidades de comprobación empírica.

Los tres criterios deben ser precisados con la delimitación del problema. Según Gomezjara y Pérez (1987), al delimitar el problema se determina el contexto en que 
la pregunta es investigada y resuelta; y el contexto en que la respuesta será validada o construida.

Además, la delimitación exige del investigador la capacidad de proveer al problema de un carácter operativo, es decir, llevarla al nivel de ejecutable el proceso de investigación. Eso, significa pensar en un tiempo, espacio y población.

Por lo tanto, hay un principio que el investigador debe tomar en cuenta: en investigación, menos, es más. La limitación de la extensión favorecerá el reconocimiento del ámbito de estudio, el encuadre teórico, la realización de una revisión exhaustiva del asunto, el dominio y su eventual resolución.

\section{Conclusiones}

- El problema es toda situación en la que hay un planteamiento inicial y una exigencia que obliga a transformarlo. Observar la realidad con creatividad a fin de encontrar problemas donde los demás no lo logran constituye el resultado de la aplicación interactiva de múltiples destrezas cognitivas; por lo mismo, un mérito científico del investigador, que es capaz de descifrar anomalías, vacíos cognoscitivos, allí, donde otros no ven o encuentran problemas. Por lo tanto, se requiere más que dominio metodológico, sin temor a equivocación, fundamentalmente hace falta talento y creatividad para problematizar y proyectar soluciones.

- La situación problemática se constituye de factores o circunstancias contradictorias de vacío cognoscitivos o de obstáculo con existencia real e independencia del sujeto; pero con afección por el que se interesa en comprenderlo y, eventualmente, resolverlo. Es el resultado de comparar el comportamiento real con el comportamiento ideal. Si ambos comportamientos son iguales, no existe contradicción o situación problemática, si, por el contrario, se presenta un estado de desigualdad, entonces hay situación problemática.

- Para determinar la situación problemática, el investigador tiene hasta tres vías. La primera asumir instrumentos estandarizados, la segunda trabajar en la contextualización de instrumentos, la tercera construir sus propios instrumentos que deben cumplir con ciertos criterios de confiablidad y validez. Cualesquiera de las tres vías son importantes y deberán concretarse en el recojo de datos, resultados que indicarán si realmente estos niños cuidan el medio ambiente. Es decir, se determina el comportamiento real, el estado actual de desarrollo, del objeto o fenómeno de investigación.

- La situación problemática pueden ser teórica o práctica. Las dificultades teóricas son vacíos de conocimiento sobre determinados factores de la realidad, 
superables con investigaciones o "reinvestigaciones" que acopien información para la producción de nuevos conocimientos descriptivos y explicativos. Las dificultades prácticas, requieren intervención o diseñó de resultados científicos fundados en teorías que, al ser aplicadas, eventualmente, permitiría la transformación del problema.

- El problema científico es la síntesis de un proceso analítico y sintético, crítico y creativo a la vez. Su adecuada formulación ocurre en simultáneo, con la fundamentación teórica, metodológica, y las posibilidades de solución. Por cuanto a mayor conocimiento y experiencia sobre el tema, mayores probabilidades de problematizar y crear soluciones. El criterio de su viabilidad es muy importante, siendo una de ellas la delimitación que favorecerá el reconocimiento del ámbito de estudio, el encuadre teórico, la realización de una revisión exhaustiva del asunto, el dominio y su eventual resolución. 


\section{Referencias}

American Psychological Association (2010). Manual de Publicaciones de la American Psychological Association (3ra.ed.). México, D.F.: Manual Moderno.

Bunge, M. (1975). La investigación científica, su estrategia y su filosofía. Barcelona, España: Ariel.

García, F. y García, L. (2005). La problematización. México. Instituto Superior de Ciencias de la Educación

Gornezjara, F. y Pérez, N. (1987). El diseño de la investigación. (2a. ed.). México: Fontamara.

Labarre, G. y Valdivia, G. (2005). Pedagogía. La Habana: Pueblo y Educación.

Lanuez, M., Martínez, M. y Pérez, V. (20 I 0). El maestro y la investigación educativa en el siglo XXI. La Habana: Pueblo y educación.

Hernández, M y Lázaro, J. (20 I0). ¿Una ciencia para enseñar Biología? La Habana: Academia.

Kerlinguer, F. y Lee, H. (2002). Investigación del comportamiento: Métodos de Investigación en Ciencias Sociales. México: McGRAW-HILL 